\title{
Effects of Au nanoparticles on thermoresponsive genipin-crosslinked gelatin hydrogels
}

\author{
Ana L. Daniel-da-Silva • Ana M. Salgueiro • Tito Trindade
}

Published online: 7 January 2013

(C) The Author(s) 2013. This article is published with open access at SpringerLink.com

\begin{abstract}
Gold gelatin hydrogel nanocomposites crosslinked with genipin have been prepared, and the effect of citrate capped Au nanoparticles (NPs) as nanofillers in the crosslinking and swelling of gelatin and release of a model drug (methylene blue) from gelatin nanocomposites have been investigated. The citrate-capped Au NPs prevented the crosslinking reaction between the gelatin and genipin and resulted in less crosslinked hydrogels. Although less crosslinked, the Au gelatin nanocomposites swelled less than the unfilled crosslinked gelatin. The gelatin composites were optically active and thermo-sensitive in a temperature range acceptable for living cells. In vitro release studies demonstrated that the irradiation of the composite gels with monochromatic green light ( $\lambda=532 \mathrm{~nm}, 100 \mathrm{~mW})$ increases the release of the encapsulated methylene blue, most likely due to the photothermal effect of Au nanoparticles. This opens the possibility to explore the application of these nanocomposites as carriers in remotely controlled light-triggered drug release.
\end{abstract}

Keywords Gold nanoparticles · Gelatin · Genipin · Biomaterials

\section{Introduction}

Recent years have witnessed growing importance of hydrogel nanocomposites for bio-applications [1,2]. These materials bring together the intrinsic functionalities of inorganic nanoparticles (NPs) and the properties of tridimensional networks offered by hydrogels. These hydrogels can be

\footnotetext{
A. L. Daniel-da-Silva $(\bowtie) \cdot$ A. M. Salgueiro $\cdot$ T. Trindade Department of Chemistry, CICECO, Aveiro Institute of Nanotechnology, University of Aveiro, 3810-193 Aveiro, Portugal

e-mail: ana.luisa@ua.pt
}

obtained from biopolymers that in controlled experimental conditions provide hydrophilicity, soft consistency, and ability to accommodate biomolecules. All these properties are attractive to promote the efficient transport of pharmaceuticals in living systems, and as such, biocompatible hydrogels have been widely used in drug delivery procedures. A recent trend in the development of hydrogels for drug delivery has been the implementation of multifunctionality, thus leading to smart drug carriers responsive to external stimuli, such as applied magnetic gradients [3,4], monochromatic light irradiation [5] or temperature effects [6]. These endeavors have relied in large extent on the use of inorganic NPs such as gold NPs that are used as functional nanofillers in the hydrogel matrix.

Gelatin is a thermoresponsive hydrogel derived from collagen that has been widely used in bio-applications, either on its own or combined with inorganic phases [7,8]. For example, gelatin composites filled with calcium phosphate have found application in tissue engineering [7] and gelatin fibers containing Ag NPs have been used in antibacterial wound-dressing materials [8]. Gelatin gels have a triple-helical structure that can be crosslinked to improve the thermal and mechanical stability of the network. Gelatin crosslinking has been achieved mainly by using chemical agents, most commonly glutaraldehyde whose major drawback is toxicity [9]. Alternatively, genipin (Fig. 1) is a chemical crosslinker, with very low toxicity, obtained from the natural occurring product geniposide that is extracted from Gardenia jasminoides fruits. Genipin has attracted great attention due to its ability to crosslink polymer chains containing primary amines, namely proteins such as gelatin [10-12] and polysaccharides such as chitosan $[10,13]$. The cytotoxicity of genipin is ca. 10,000 times lower than that of glutaraldehyde [14] and therefore genipin is extremely valuable in the development of biocompatible materials. 


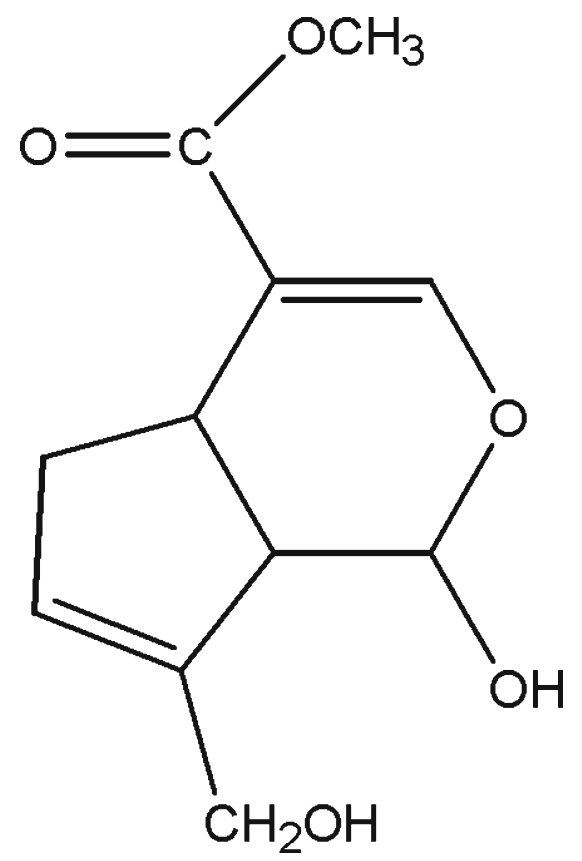

Fig. 1 Chemical structure of genipin

The recent interest in Au hydrogel nanocomposites for biomedical applications relies mostly on their optical properties due to the plasmonic behavior of Au NPs dispersed in the matrix. For $\mathrm{Au} \mathrm{NPs}$, the surface plasmon resonance (SPR) band is located in the visible and is sensitive to several parameters, including particle size and shape, dielectric constant of the dispersing medium [15]. In addition, $\mathrm{Au}$ NPs act as heat dissipators by absorbing light at a frequency matching that one of the SPR band. This effect has been exploited to induce localized temperature gradients that can be used in photothermal therapy [16] and to trigger the release of encapsulated drugs from thermosensitive hydrogels [5,17]. Examples of Au hydrogel nanocomposites are those of chitosan [17,18] and alginate [19]. Although there are a few reports on the preparation of Au gelatin nanocomposites [20,21], the effect of light-irradiated Au NPs on the release properties of the hydrogel has not been investigated. Moreover, there are no reports on the use of genipin for preparing crosslinked $\mathrm{Au}$ gelatin nanocomposites, despite its relevance in the context of new formulations for biocompatible hydrogels.

In the sequence of our current work on the development of hydrogel nanocomposites for controlled release [22,23], we report here the preparation of genipin-crosslinked $\mathrm{Au}$ gelatin nanocomposites. This work aims to investigate the effect of colloidal gold nanoparticles on the crosslinking, thermal, swelling, and release properties of genipincrosslinked gelatin hydrogels. Selected hydrogel nanocomposites were then tested for in vitro release of methylene blue, a commonly used model, with and without light irradiation of the gels.

\section{Materials and methods}

Materials

Porcine gelatin (type A, 300 bloom, Sigma-Aldrich), genipin $\left(\mathrm{C}_{11} \mathrm{H}_{14} \mathrm{O}_{5}\right)(98 \%$, Chengdu King-tiger Pharm-chem. Tech.Co., Ltd), tetrachloroauric acid $\left(\mathrm{HAuCl}_{4} \cdot 3 \mathrm{H}_{2} \mathrm{O}\right)$ (99.9\%, Sigma-aldrich), trisodium citrate dihydrate (HOC $\left.(\mathrm{COONa})\left(\mathrm{CH}_{2} \mathrm{COONa}\right)_{2} \cdot 2 \mathrm{H}_{2} \mathrm{O}\right)(99 \%$, Sigma-Aldrich), methylene blue $\left(\left(\mathrm{C}_{16} \mathrm{H}_{18} \mathrm{ClN}_{3} \mathrm{~S}\right)\right.$ (Riedel-de-Häen), and phosphatebuffered saline (PBS) solution (pH7.4, Sigma-Aldrich) were used as received without any further purification.

\section{Synthesis of Au nanoparticles}

Gold NPs were prepared by reduction of a gold(III) complex using sodium citrate as reducing agent. Typically, $3.76 \mathrm{ml}$ of an aqueous solution of sodium citrate $(96.8 \mathrm{mM})$ was added to a mixture of $95 \mathrm{ml}$ of ultrapure water and $3.7 \mathrm{ml}$ of $\mathrm{HAuCl}_{4} \cdot 3 \mathrm{H}_{2} \mathrm{O}$ aqueous solution $(12.95 \mathrm{mM})$ at $80{ }^{\circ} \mathrm{C}$, under vigorous stirring and reflux, and allowed to react over $1 \mathrm{~h}$. A deep-red hydrosol was obtained, which indicated the formation of the gold colloid.

\section{Preparation of $\mathrm{Au} /$ gelatin nanocomposites}

The nanocomposites were prepared by blending the Au NPs with the gelatin as follows. The colloidal $\mathrm{Au}(1.25,2.5$, and $3.75 \mathrm{ml}$ ) was added to an aqueous solution of gelatin previously prepared, at $45^{\circ} \mathrm{C}$. The volume of water was adjusted to perform a total of $15 \mathrm{~mL}$ and the gelatin concentration was $15 \mathrm{gL}^{-1}$. After achieving a homogenous dispersion of the nanoparticles, $1.25 \mathrm{ml}$ of the selected model drug, methylene blue $0.3 \mathrm{gL}^{-1}$, was added. The mixture was cool down until $40{ }^{\circ} \mathrm{C}$ and $2.25 \mathrm{ml}$ of genipin solution $4 \mathrm{gL}^{-1}$ was added and stirred for $30 \mathrm{~min}$. Afterwards, $2.5 \mathrm{ml}$ of the composite mixture was transferred to a cylindrical glass vial (Ø $17 \mathrm{~mm})$ which was sealed and left for incubation for $48 \mathrm{~h}$ at $25^{\circ} \mathrm{C}$. The content of genipin in the composites was $0.4 \mathrm{wt} \%$, related to the gelatin. The Au NPs load in the composites was calculated assuming complete conversion of the gold complex into metal Au NPs and was found to be 52, 104, and $156 \mathrm{ppm}$. After incubation, the nanocomposite discs presented a dark blue appearance which is related to the level of crosslinking of gelatin. The gel samples were frozen at $-5^{\circ} \mathrm{C}$ for $24 \mathrm{~h}$ and lyophilized. The final freeze dried discs had approximately $15 \mathrm{~mm}$ diameter and $8 \mathrm{~mm}$ height.

Swelling studies

The swelling measurements were carried out by immersion of lyophilized hydrogel discs in $50 \mathrm{ml}$ PBS $0.01 \mathrm{M} \mathrm{pH} 7.4$ at $37{ }^{\circ} \mathrm{C}$. At the required intervals of time, the samples were removed from the solution and wiped with filter paper to 
remove the excess of water before being weighted. The swelling ratio $(Q)$ was calculated from Eq. 1:

$Q=\frac{W_{\mathrm{s}}-W_{\mathrm{d}}}{W_{\mathrm{d}}}$

where $W_{\mathrm{d}}$ and $W_{\mathrm{s}}$ are the weight of the lyophilized and swollen gel, respectively. The equilibrium swelling ratio ( $\left.Q_{\text {equil }}\right)$ was determined at the point the hydrated gels achieved a constant weight value. The swelling experiments were performed in triplicate.

\section{Materials characterization}

FTIR analysis Fourier transform infrared (FTIR) spectra of the lyophilized gelatin and gelatin nanocomposites were collected using a spectrometer Mattson 7000 coupled to a horizontal attenuated total reflectance (ATR) cell, accumulating 256 scans and using a resolution of $4 \mathrm{~cm}^{-1}$. FTIR spectrum of the Au nanoparticles was collected after drying the colloidal suspension in a $\mathrm{KBr}$ pellet. The spectrum was collected with 256 scans and $4 \mathrm{~cm}^{-1}$ resolution.

Transmission electron microscopy Transmission electron microscopy (TEM) analysis of Au nanoparticles was performed using a transmission electron microscope JEOL $200 \mathrm{CX}$ operating at an accelerating voltage of $30 \mathrm{kV}$. Samples for TEM analysis were prepared by evaporating dilute suspensions of the nanoparticles on a copper grid coated with an amorphous carbon film.

Differential scanning calorimetry The gel-sol transitions of hydrogels were determined by differential scanning calorimetry (DSC) using a Shimadzu DSC-50 calorimeter. With sample masses of ca. $25 \mathrm{mg}, 30-\mu \mathrm{L}$ aluminum pans were used. Samples were heated from 25 to $80{ }^{\circ} \mathrm{C}$ at $2{ }^{\circ} \mathrm{Cmin}^{-1}$. An empty pan was used as reference.

Zeta potential measurements The surface charge of the $\mathrm{Au}$ NPs was assessed by zeta potential measurements, using a Zetasizer Nanoseries instrument from Malvern Instruments (UK).

$U V$-vis spectrophotometry The optical properties of $\mathrm{Au}$ NPs and gelatin $\mathrm{Au}$ nanocomposites were investigated by UV-vis analysis of aliquots of the samples. A Jasco V 560 UV/Vis spectrophotometer (Jasco Inc., USA) was used for recording the UV/vis absorption spectra of the aliquots.

In vitro MB release studies

Methylene blue (MB) was used as a model drug and was loaded during the stage of the preparation of the nanocomposites as described above. MB has been used as a model drug namely because it is a water-soluble dye that allows an immediate visual inspection of the test. The release experiments were performed with and without laser irradiation of the samples.

The release experiments carried out without laser irradiation were performed in a thermostatic orbital shaker KS 4000I Control from IKA at the physiological temperature $37{ }^{\circ} \mathrm{C}$ and $120 \mathrm{rpm}$. A lyophilized disc was introduced in a glass beaker containing $50 \mathrm{ml}$ PBS $0.01 \mathrm{M} \mathrm{pH} 7.4$ and $0.05 \%(w / v)$ sodium azide as preserving agent. After predetermined intervals, $1.0 \mathrm{ml}$ of the release medium was drawn and analyzed by UV-vis spectroscopy $(\lambda=663 \mathrm{~nm})$ to determine the amount of MB released at each time point and replaced by $1 \mathrm{ml}$ of fresh PBS to maintain the original volume. The cumulative released fraction at time $t\left(m_{t} / \mathrm{m}_{0}\right)$ was calculated using Eq. 2:

$\frac{m_{t}}{m_{0}}=\frac{50 \times C_{\mathrm{n}}+\sum_{i=0}^{n-1} C_{i}}{m_{0}}$

where $m_{t}$ is the cumulative mass of MB released at time $t, m_{0}$ is the original mass of MB loaded, $C_{i}$ is the mass concentration of MB (per milliliter) of the aliquot, $C_{\mathrm{n}}$ is the mass concentration of MB (per milliliter) of the aliquot at time $t$, and $n$ is the total number of aliquots extracted until time $t$.

The release experiments carried out with laser irradiation were performed at room temperature (ca. $11{ }^{\circ} \mathrm{C}$ ). In a typical experiment, the crosslinked gelatines containing methylene blue were prepared in a glass tube $\left(\varnothing_{\text {int }}=0.6 \mathrm{~cm}\right)$, following the procedure described above. The final volume of the gelatin samples was $0.25 \mathrm{~mL}$ per tube. A volume of $0.7 \mathrm{~mL}$ of PBS $0.01 \mathrm{M} \mathrm{pH7.4}$ was added to each tube. The gelatines were irradiated with the laser spot positioned immediately below the interface gel/PBS using a $\mathrm{CW}$ diode pumped solid state laser $(\lambda=532 \mathrm{~nm}, 100 \mathrm{~mW})$ located at a distance of $10 \mathrm{~cm}$ from the sample. After irradiation, the PBS solution was analyzed by UV-vis spectroscopy $(\lambda=$ $663 \mathrm{~nm}$ ) to determine the amount of MB released. For comparison, release tests were also performed in identical conditions, in the absence of laser irradiation.

\section{Results and discussion}

\section{Characterization of Au colloid}

Gold NPs were prepared by reduction of a gold(III) complex in aqueous medium using sodium citrate as reducing and stabilizing agent. The resulting aqueous $\mathrm{Au}$ colloid 

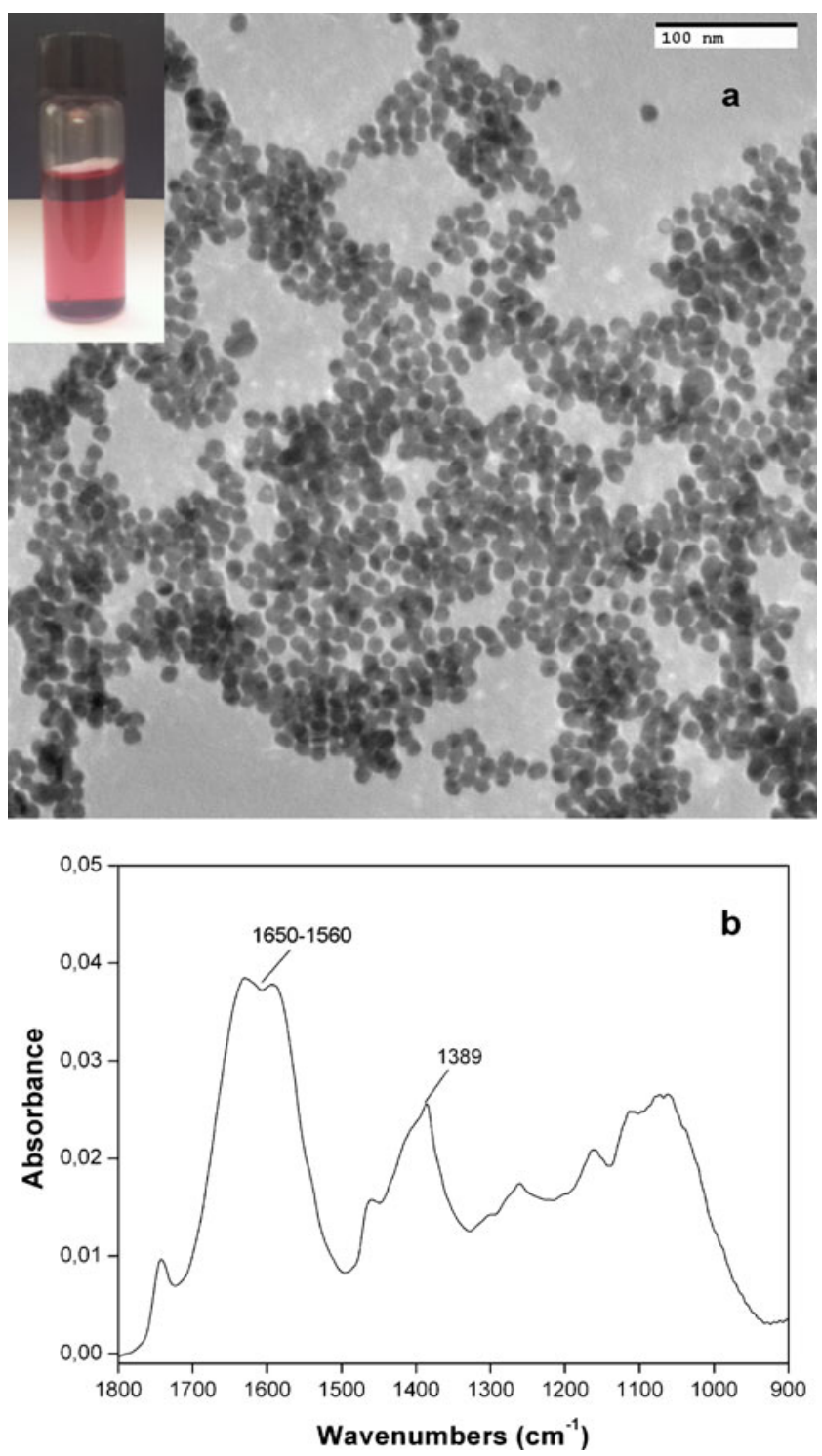

Fig. 2 a TEM micrograph and b FTIR spectrum of colloidal Au NPs used as dispersed phase

exhibited a red color (Fig. 2a, inset) due to the SPR band peaked about $520 \mathrm{~nm}$. The average particle size for Au NPs was found to be $10 \pm 2 \mathrm{~nm}$ as determined by transmission electron microscopy (Fig. 2a).

Gold colloids as prepared above are stable because surface chemisorbed citrate ions impart a net negative charge to the particles causing electrostatic repulsion. This was confirmed by zeta potential measurements $(-40.1 \pm 0.8 \mathrm{mV}, \mathrm{pH}$ 6.8) performed on the aqueous Au colloids. FTIR spectrum of Au colloid (Fig. 2b) shows two broad bands, one in the region $1,560-1,650 \mathrm{~cm}^{-1}$ and the other centered at $1,389 \mathrm{~cm}^{-1}$ corresponding, respectively, to the asymmetric and symmetric stretching vibrations of carboxylate groups $[24,25]$, thus confirming that the Au NPs are capped with citrate anions.
Chemical and thermal characterization of $\mathrm{Au}$ and gelatin nanocomposites

The ATR-FTIR spectrum of the non-crosslinked gelatin (Fig. 3) shows the vibrations amide I and amide II characteristic from polypeptides. The band in the amide I region is centered at $1,629 \mathrm{~cm}^{-1}$ and corresponds to the $\mathrm{C}=\mathrm{O}$ stretching vibration in the amide group coupled to the in-phase bending of the $\mathrm{N}-\mathrm{H}$ bond and the $\mathrm{C}-\mathrm{N}$ stretching vibration [26]. The band in the amide II region that corresponds to the $\mathrm{N}-\mathrm{H}$ bending vibration coupled to stretching $\mathrm{C}-\mathrm{N}$ vibrations was identified at $1,524 \mathrm{~cm}^{-1}$. The spectrum of genipincrosslinked gelatin is very similar to that of non-crosslinked sample. However, the amide II band is slightly less intense than in the non-crosslinked gelatin. This indicates the reduction of the number of primary amine groups due to the reaction with genipin molecules [27].

The ATR-FTIR spectra of the genipin-crosslinked $\mathrm{Au}$ gelatin nanocomposites are shown in Fig. 3. These spectra are dominated by the vibrational bands ascribed to gelatin due to the minor amount of Au NPs present in the composite. Nevertheless, a small shift of the amide II band from $1,524 \mathrm{~cm}^{-1}$, in the unfilled crosslinked gelatin, to $1,536 \mathrm{~cm}^{-1}$ in the composites is observed in Fig. 3. This band shift suggests that Au NPs interact with amine groups from gelatin, most likely via electrostatic interactions between carboxylate and protonated amine groups. At the $\mathrm{pH}$ of the composite mixture (pH5.3), the amine groups from gelatin are protonated and can interact electrostatically with citrate groups capping the Au NPs. These results are in agreement with previous observations that report changes in the IR spectra for the amide II region of different proteins, after the addition of citrate-stabilized Au colloidal nanoparticles [28]. Other workers have proven the electrostatic

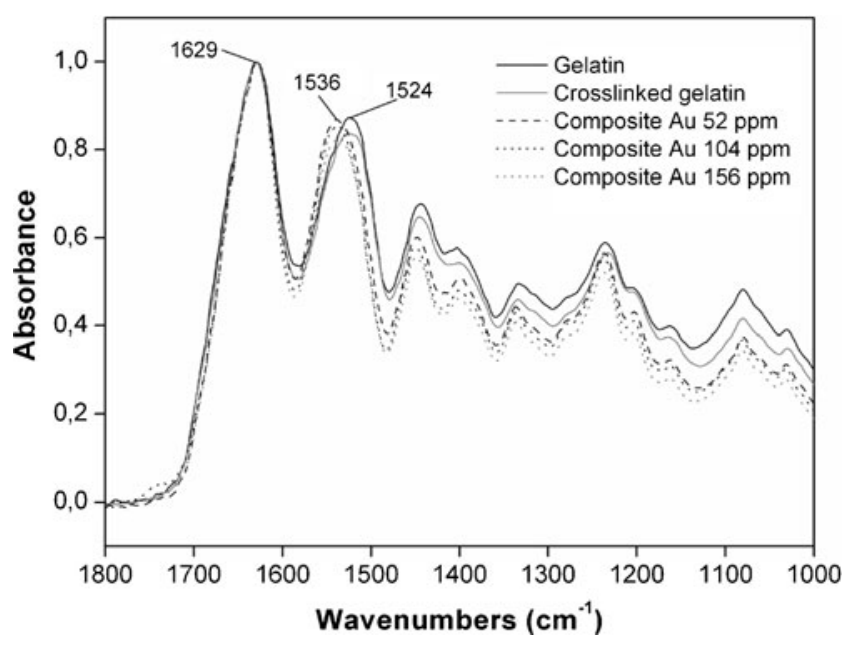

Fig. 3 FTIR-ATR spectra of uncrosslinked gelatin, genipincrosslinked gelatin, and $\mathrm{Au}$ nanocomposites. The intensity of spectra was normalized in relation to the amide I vibration band 
binding of bovine serum albumin to citrate-capped gold NPs due to interactions between carboxylate and protonated amine groups using non-spectroscopic techniques [29].

The addition of $\mathrm{Au}$ NPs at a concentration of $52 \mathrm{ppm}$ resulted also in the increase of the intensity of the Amide II band in the nanocomposite when compared to the unfilled crosslinked gelatin. This suggests that, due to the interaction of citrate/amine groups, the citrate-capped Au NPs prevent the reaction between the gelatin and genipin, hence restraining the reticulation of gelatin. This is in agreement with the observed decrease of the amide II band for increasing amounts of Au NPs in the nanocomposites (Fig. 3). In fact, similar effects of citrate-capped Au NPs on the chemical reactivity of the amino groups of proteins have been reported for the glycation by fructose [24].

The DSC thermograms of genipin-crosslinked gelatin and derived $\mathrm{Au}$ nanocomposites (Fig. 4) show a broad endothermic peak that corresponds to the denaturation (helix-to-coil transition) of the gelatin [11]. The values for the denaturation temperature $\left(T_{\mathrm{D}}\right)$, defined here as the temperature of the main peak, are depicted in Table 1. For the neat hydrogel, $T_{\mathrm{D}}$ is $78.9^{\circ} \mathrm{C}$ and then decreases by addition of $\mathrm{Au}$ NPs to the hydrogel, reaching the value $65.1{ }^{\circ} \mathrm{C}$ for the nanocomposite with $156 \mathrm{ppm}$ Au content. Adding Au NPs also resulted in a slight decrease of the area of the endothermic peak, which is directly related to the denaturation enthalpy $\left(\Delta H_{\mathrm{D}}\right)$ (Table 1$)$.

The decrease of $T_{D}$ might be ascribed to reduction of covalent crosslinking of the hydrogels, since an increased extent of crosslinking of gelatin implies higher $T_{\mathrm{D}}$ [11] Thus, the DSC results indicate that the incorporation of $\mathrm{Au}$ NPs restrain the formation of covalent crosslinks and are in agreement with the FTIR spectroscopic data presented above. Noteworthy, these observations demonstrate that

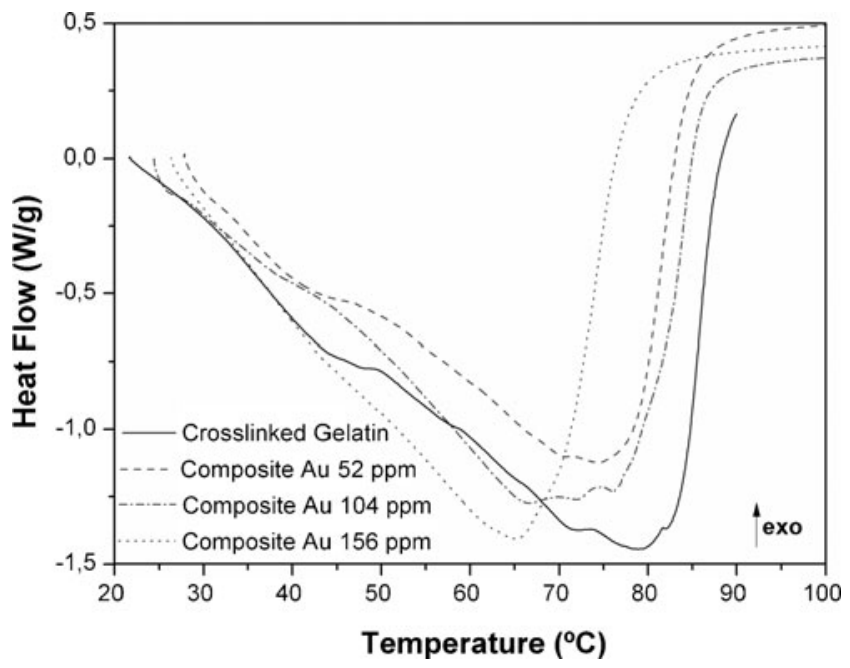

Fig. 4 DSC thermograms of genipin-crosslinked gelatin and derived Au nanocomposites
Table 1 Denaturation temperature $\left(T_{\mathrm{D}}\right)$ and enthalpy $\left(\Delta H_{\mathrm{D}}\right)$ of genipin crosslinked gelatin hydrogels and Au nanocomposites calculated from DSC experiments

\begin{tabular}{lcc} 
Au NPs (ppm) & $T_{\mathrm{D}}\left({ }^{\circ} \mathrm{C}\right)$ & $\Delta H_{\mathrm{D}}(\mathrm{kJ} / \mathrm{g})$ \\
\hline 0 & 78.9 & -2.0 \\
52 & 74.5 & -1.7 \\
104 & 69.2 & -1.8 \\
156 & 65.1 & -1.6 \\
\hline
\end{tabular}

the nanocomposites are thermo-sensitive in the $37-45{ }^{\circ} \mathrm{C}$ temperature range, i.e., the temperature range acceptable for living cells. Therefore, these composites have potential for the release of encapsulated molecules triggered by thermal stimuli.

Optical properties of the nanocomposites

The visible spectrum of the aqueous Au colloid showed a well-defined SPR band centered at $523 \mathrm{~nm}$ (Fig. 5a), characteristic of nearly monodispersed Au NPs, whose
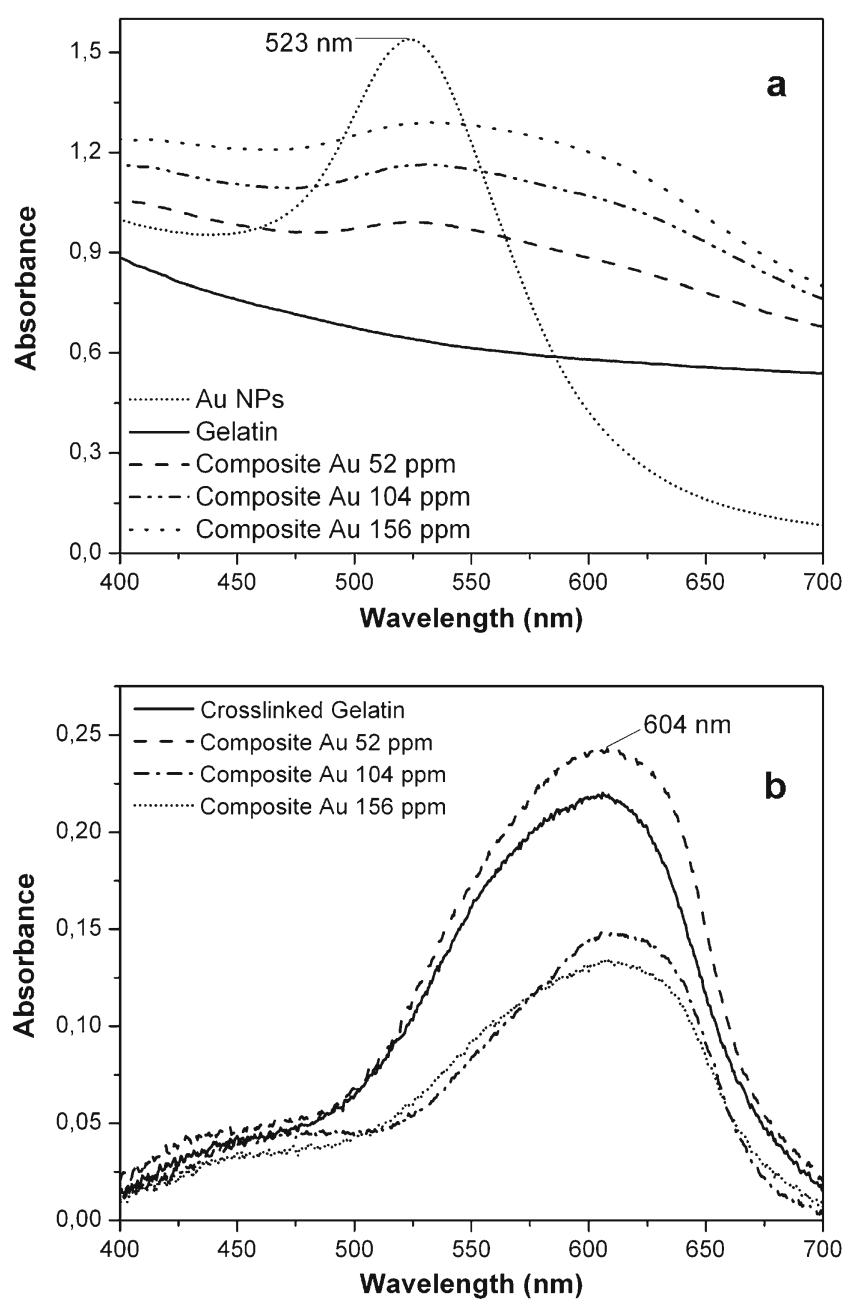

Fig. 5 Absorption spectra of the gelatin nanocomposites with variable $\mathrm{Au}$ NPs content $\mathbf{a}$ before reticulation with genipin and $\mathbf{b}$ reticulated with genipin 
morphological characteristics are shown in Fig. 2. On the other hand, the visible spectra of gelatin $\mathrm{Au}$ composites prior reticulation with genipin (Fig. 5a) display a broader band and a shoulder at ca. $600 \mathrm{~nm}$, the latter becoming more pronounced for increasing $\mathrm{Au}$ content. The observation of a shoulder extending to higher wavelength in relation to the SPR band of the original colloid is an indication of Au NPs aggregation, probably mediated by the biopolymer chains, due to interparticle plasmon coupling. This behavior apparently contrasts with the stabilizing effect previously reported for gelatin [20]. However, it should be noted that this stabilizing effect was reported for in situ prepared Au NPs, using gelatin as a reducing and stabilizing agent, thus in the absence of citrate anions [20]. Thus, it might be inferred that the Au NPs aggregation observed in this case arises from the interaction between the gelatin and the citrate capping. These results are in line with previous observations indicating that the adsorption of proteins onto the surface of citrate-capped Au NPs induce their aggregation [29]. At the temperature used for preparing the composites $\left(45^{\circ} \mathrm{C}\right)$, the gelatin polypeptide chains exist predominantly in the form of flexible, unfold coils in solution, and the adsorption of these chains onto the Au NPs surface might also be expectable, thus promoting the NPs aggregation.

For the case of crosslinked gelatin, the optical spectra also evidence the effect of genipin. In fact, these hydrogels exhibit a characteristic blue color that arises from products of the reaction of genipin with amino acids of gelatin $[27,30]$. The blue pigments are most likely formed through the oxygen radical-induced polymerization and dehydrogenation of intermediate compounds [31]. As a result, the optical spectra of the crosslinked gelatin shows a broad band centered at $600 \mathrm{~nm}$ that for the nanocomposites spectra probably have some contribution from the broadening of the SPR band of Au NPs. Figure 5b shows that the overall trend in the composites is the decay of the absorbance peaks at ca. $600 \mathrm{~nm}$ with increasing amount of Au NPs. This indicates that less genipin has reacted in the presence of citrate-capped Au NPs and is well in line with FTIR and DSC observations. Crosslinked composites containing $52 \mathrm{ppm} \mathrm{Au}$ NPs show however a slight increase of the absorbance peak at $600 \mathrm{~nm}$ in relation to the unfilled crosslinked gelatin that contrasts with the overall trend. This can be ascribed to the contribution of the aggregation of the $\mathrm{Au}$ NPs that leads to an increase of the absorbance at these wavelengths, as seen in Fig. 5a.

\section{Swelling properties}

Figure 6 displays the swelling ratio in function of time, for genipin-crosslinked gelatin hydrogels in PBS, and for variable Au NPs load. The unfilled hydrogels swelled smoothly over a period of $60 \mathrm{~h}$, until reaching the equilibrium

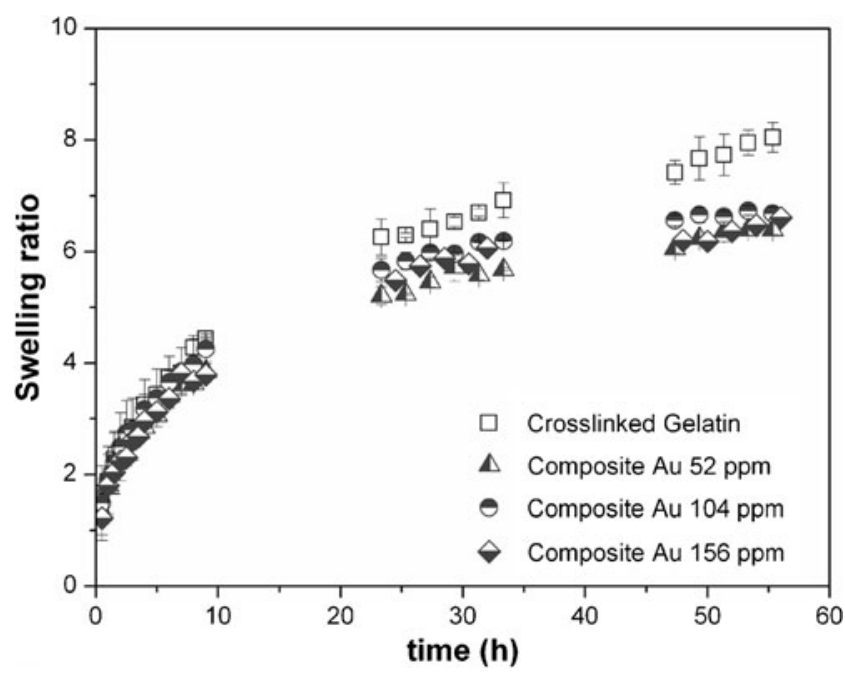

Fig. 6 Swelling ratio $(Q)$ genipin of crosslinked gelatin and derived Au nanocomposites in PBS at $37^{\circ} \mathrm{C}$ as a function of time

swelling ratio $\left(Q_{\text {equil }}\right)$ of $8.1 \pm 0.3$. The Au composite hydrogels swelled slightly slower than the unfilled gelatin and the incorporation of Au NPs resulted in a decrease of $Q_{\text {equil }}$ from 8.1 to ca. 6.5 , regardless the Au NPs content.

Gelatin swelling usually decreases with the extension of crosslinking $[12,32,33]$. The FTIR and DSC results presented above have shown a decrease on crosslinking when $\mathrm{Au}$ NPs have been added to the hydrogel. Therefore, more swelling would be expected for these nanocomposites as compared to unfilled gelatin. Indeed, the opposite effect was observed, which indicates that water diffusion into the gel was limited by the Au NPs dispersed in the network. A similar effect has been reported for montmorillonite (MMT) gelatin nanocomposites [34]. Although MMT hinders the formation of chemical crosslinkages, MMT gelatin nanocomposites crosslinked with dextran dialdehyde has been described as swelling less than the unfilled crosslinked gelatin [34]. The reduction on swelling with MMT content was ascribed to a barrier effect of the MMT NPs to the diffusion of solvent molecules into the gelatin [34].

In vitro $\mathrm{MB}$ release

In order to assess the potential of the nanocomposites for controlled release procedures, hydrogels with the highest $\mathrm{Au}$ NPs content (156 ppm) have been selected for in vitro MB release studies. The amount of MB released was monitored by measuring the absorbance of the release medium at $663 \mathrm{~nm}$, i.e., at the wavelength of maximum absorbance for MB. The release experiments were carried out with and without laser irradiation $(\lambda=532 \mathrm{~nm})$ of the samples.

Figure 7a displays the MB release profiles in PBS from the genipin-crosslinked gelatin and the Au nanocomposite, at $37{ }^{\circ} \mathrm{C}$, in absence of laser irradiation. Both hydrogels 

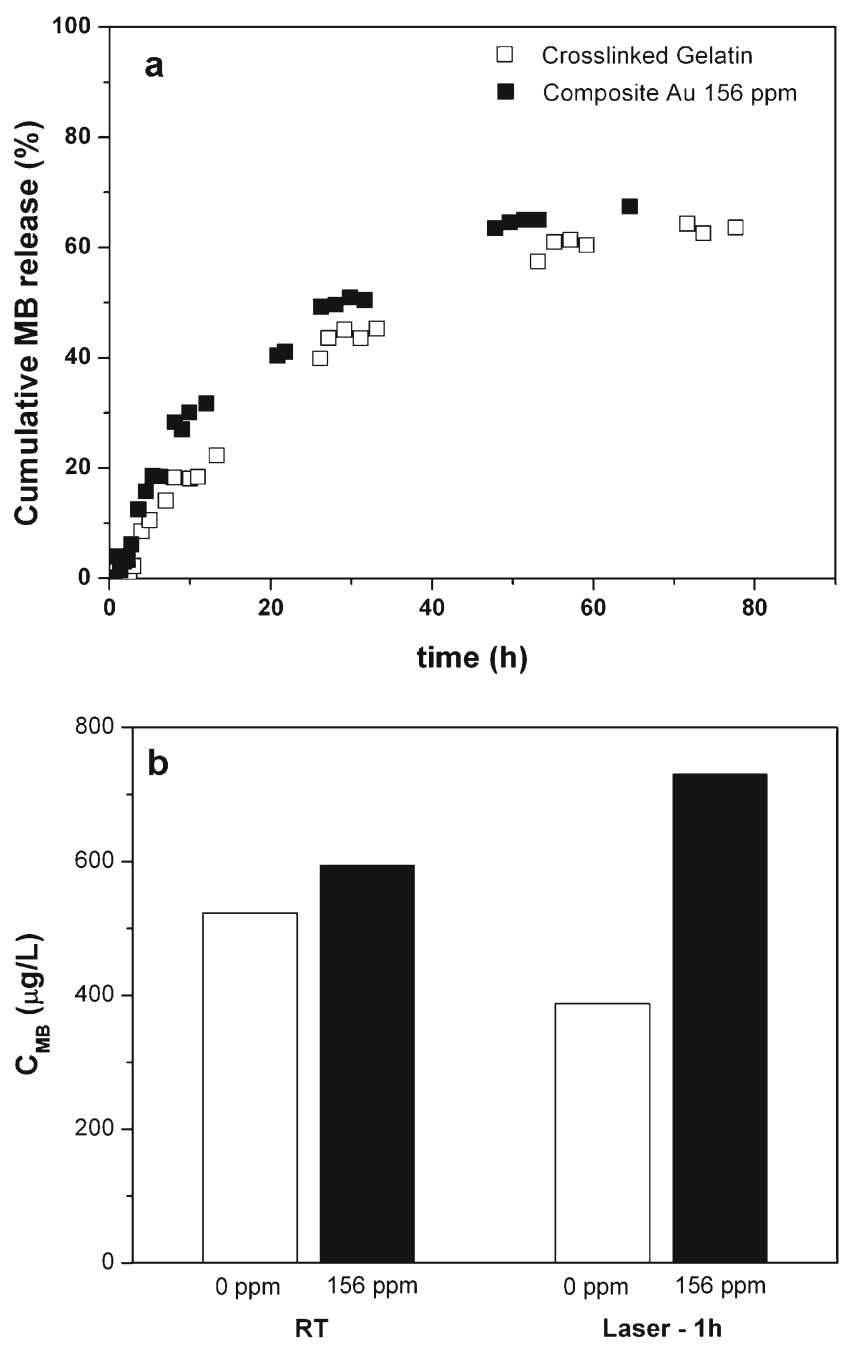

Fig. 7 In vitro MB release in PBS from unfilled crosslinked gelatin $(0 \mathrm{ppm})$ and derived Au nanocomposites (156 ppm): a at $37^{\circ} \mathrm{C}$ without irradiation, as function of time; $\mathbf{b}$ at RT, with and without exposure to green light $(\lambda=532 \mathrm{~nm})$ after $1 \mathrm{~h}$

exhibited a sustainable MB release for a period of $70 \mathrm{~h}$. Figure 7a shows a profile in which there is a fast release at an initial stage, followed by a slower rate until a steady concentration is achieved. The release profiles are identical in the first $10 \mathrm{~h}$, and minor differences are observed afterwards. Apparently, the decrease of the swelling ratio in the composites does not affect the MB release profile. Also it appears that the Au NPs do not limit the release of MB from the gelatin matrix, conversely to the effect reported for the MB release from $\mathrm{Au}$ nanocomposites prepared with silicone elastomers [35] and k-carrageenan [36]. The decrease of MB release in these composites was ascribe to the interaction between $\mathrm{Au}$ NPs and MB, most probably by chemisorption of MB onto Au NPs surface via sulfur atoms [37], that limits the diffusion of MB from the polymer matrix. The absence of this effect on the gelatin composites supports the assumption that the gelatin chains are strongly adsorbed onto the Au NPs surface, as discussed above. In this case, the gelatin polypeptide chains might enfold the Au NPs and thus prevent the sorption of MB molecules onto the NPs surfaces.

Figure $7 \mathrm{~b}$ shows the amount of MB released to the surrounding medium after exposure of the gels to the laser light $(\lambda=532 \mathrm{~nm})$ for a period of $1 \mathrm{~h}$. Under exposure to the green laser, the MB released from the Au composite (156 ppm) is approximately twice than in the unfilled gelatin. For comparison, the MB released was also quantified in identical experimental conditions, without exposing the gels to the green light. In absence of laser exposure, both gels (blank and $\mathrm{Au}$ composite) release equivalent amounts of MB. This is in agreement with the results above presented (Fig. 7a) for the release experiments performed at physiological temperature without irradiation. The results show an increment of the MB released from the Au composites in $25 \%$ after exposed to the green light, an effect that might be ascribed to the photothermal conversion of Au nanoparticles. Indeed, the wavelength of the incident light $(\lambda=532 \mathrm{~nm})$ matches the maximum absorption of the SPR band of the Au nanoparticles, and therefore, it is expected that the absorption of this light by the nanoparticles will increase locally the temperature. Since genipin-crosslinked gelatin is sensitive to temperature, as confirmed by DSC results (Fig. 4), it is expected that this heating will induce local transformations in the gel network, thus promoting the release of the encapsulated MB molecules, as observed. It should be noted that, in opposition to the behavior observed in the Au composites, the irradiation of the blank hydrogel seems to decrease the amount of MB released (Fig. 7b), most probably due to photobleaching of some of the MB molecules trapped in the gelatin matrix, as previously reported [38].

\section{Conclusions}

The effect of colloidal Au NPs as nanofillers in the crosslinking and swelling of genipin-crosslinked gelatin hydrogels and methylene blue (MB) release from Au gelatin nanocomposites have been investigated. The citrate-capped $\mathrm{Au}$ NPs prevent the reaction between the gelatin and genipin and hinder the formation of the gelatin crosslinking network, most probably due to the interaction of carboxylate groups with protonated amine groups from gelatin residues. Although less crosslinked, the $\mathrm{Au}$ gelatin nanocomposites swell less than the unfilled crosslinked gelatin, which suggest that Au NPs may act as barrier for the diffusion of solvent molecules into the gel network. Nevertheless, the incorporation of Au NPs did not affect the MB release from gelatin in isothermal conditions. The gelatin composites were thermo-sensitive at the physiological temperatures $\left(37-45^{\circ} \mathrm{C}\right)$ and revealed optical features from $\mathrm{Au}$ NPs. The irradiation of the composite gels with monochromatic 
green light $(\lambda=532 \mathrm{~nm})$ in the range of the Au NPs SPR band promoted the release of the encapsulated methylene blue, most probably due to the photothermal effect of $\mathrm{Au}$ nanoparticles. Thus, applications of these nanocomposites as carriers in light-triggered drug release can be envisaged.

Acknowledgments The authors acknowledge FCT - Fundação para a Ciência e Tecnologia (ERA-Eula/0003/2009, Pest-C/CTM/LA0011/ 2011), FSE, and POPH for funding. We thank the RNME (National Electronic Microscopy Network) for TEM images. The authors are very grateful to M.Sc. M.C. Azevedo and Dr. A.V. Girão (University of Aveiro, Chemistry Department) for technical support and to M.Sc. Marco Peres and Prof. Jorge Soares (University of Aveiro, Physics Department) for their assistance in laser experiments.

Open Access This article is distributed under the terms of the Creative Commons Attribution License which permits any use, distribution, and reproduction in any medium, provided the original author(s) and the source are credited.

\section{References}

1. Trindade T, Daniel-Silva AL (2011) Nanocomposite particles for bio-applications: materials and bio-interfaces. Pan Stanford Publishing Pte. Ltd, Singapore

2. Schexnailder P, Schmidt G (2009) Nanocomposite polymer hydrogels. Colloid Polym Sci 287:1-11

3. Satarkar NS, Hilt JZ (2008) Magnetic hydrogel nanocomposites for remote controlled pulsatile drug release. J Control Release 130:246-251

4. Brazel CS (2009) Magnetothermally-responsive nanomaterials: combining magnetic nanostructures and thermally-sensitive polymers for triggered drug release. Pharm Res 26:644-656

5. Kang H, Trondoli AC, Zhu G, Chen Y, Chang Y-J, Liu H, Huang Y-F, Zhang X, Tan W (2011) Near-infrared light-responsive coreshell nanogels for targeted drug delivery. ACS Nano 5:5094-5099

6. Daniel-da-Silva AL, Ferreira L, Gil AM, Trindade T (2011) Synthesis and swelling behavior of temperature responsive $\mathrm{K}$ carrageenan nanogels. J Colloid Interf Sci 355:512-517

7. Chen K-Y, Yao C-H (2011) Repair of bone defects with gelatinbased composites: a review. Biomedicine 1:29-32

8. Rujitanaroj P-O, Pimpha N, Supaphol P (2008) Wound-dressing materials with antibacterial activity from electrospun gelatin fiber mats containing silver nanoparticles. Polymer 49:4723-4732

9. Sisson K, Zhang C, Farach-Carson MC, Chase DB, Rabolt JF (2009) Evaluation of cross-linking methods for electrospun gelatin on cell growth and viability. Biomacromolecules 10:1675-1680

10. Chiono V, Pulieri E, Vozzi G, Ciardelli G, Ahluwalia A, Giusti P (2008) Genipin-crosslinked chitosan/gelatin blends for biomedical applications. J Mater Sci Mater Med 19:889-898

11. Bigi A, Cojazzi G, Panzavolta S, Roveri N, Rubini K (2002) Stabilization of gelatin films by crosslinking with genipin. Biomaterials 23:4827-4832

12. Yao C-H, Liu B-S, Chang C-J, Hsu H-S, Chen Y-S (2004) Preparation of networks of gelatin and genipin as degradable biomaterials. Mater Chem Phys 83:204-208

13. Muzzarelli RAA (2009) Genipin-crosslinked chitosan hydrogels as biomedical and pharmaceutical aids. Carbohyd Polym 77:1-9

14. Mi FL, Tan YC, Liang HC, Huang RN, Sung HW (2001) In vitro evaluation of a chitosan membrane crosslinked with genipin. J Biomater Sci, Polym Ed 12:835-850

15. Daniel M-C, Astruc D (2004) Gold nanoparticles: assembly, supramolecular chemistry, quantum-size-related properties, and applications toward biology, catalysis, and nanotechnology. Chem Rev 104:293-346

16. Pissuwan D, Cortie CH, Valenzuela SM, Cortie MB (2007) Gold nanosphere-antibody conjugates for hyperthermal therapeutic applications. Gold Bull 40:121-129

17. Choi WI, Kim JY, Kang C, Byeon CC, Kim YH, Tae G (2011) Tumor regression in vivo by photothermal therapy based on goldnanorod-loaded, functional nanocarriers. ACS Nano 5:1995-2003

18. Matteini P, Ratto F, Rossi F, Centi S, Dei L, Pini R (2010) Chitosan films doped with gold nanorods as laser-activatable hybrid bioadhesives. Adv Mater 22:4313-4316

19. Lim SY, Lee JS, Park CB (2010) In Situ growth of gold nanoparticles by enzymatic glucose oxidation within alginate gel matrix. Biotechnol Bioeng 105:210-214

20. Zhang J-J, Gu M-M, Zheng T-T, Zhu J-J (2009) Synthesis of gelatin-stabilized gold nanoparticles and assembly of carboxylic single-walled carbon nanotubes/Au composites for cytosensing and drug uptake. Anal Chem 81:6641-6648

21. Neupane MP, Park IS, Bae TS, Yin HK, Uo M, Watari F (2011) Titania nanotubes supported gelatin stabilized gold nanoparticles for medical implants. J Mater Chem 21:12078-12082

22. Daniel-da-Silva AL, Moreira J, Neto R, Estrada AC, Gil AM, Trindade T (2012) Impact of magnetic nanofillers in the swelling and release properties of $\mathrm{K}$-carrageenan hydrogel nanocomposites. Carbohyd Polym 87:328-335

23. Daniel-da-Silva AL, Fateixa S, Guiomar AJ, Costa BFO, Silva NJO, Trindade T, Goodfellow BJ, Gil AM (2009) Biofunctionalized magnetic hydrogel nanospheres of magnetite and $\kappa$-carrageenan. Nanotechnology 20:355602

24. Singha S, Dasgupta A, Kr DH (2011) Gold nanoparticle induces masking of amines and some therapeutic implications. J Nanosci Nanotechnol 11:7744-7752

25. Coleman MM, Lee JY, Painter PC (1990) Acid salts and the structure of ionomers. Macromolecules 23:2339-2345

26. Hashim DM, Man YBC, Norakasha R, Shuhaimi M, Salmah Y, Syahariza ZA (2010) Potential use of Fourier transform infrared spectroscopy for differentiation of bovine and porcine gelatins. Food Chem 118:856-860

27. Butler MF, Y-F NG, Pudney PDA (2003) Mechanism and kinetics of the crosslinking reaction between biopolymers containing primary amine groups and genipin. J Polym Sci, Part A: Polym Chem 41:3941-3953

28. Brewer SH, Glomm WR, Johnson MC, Knag MK, Franzen S (2005) Probing BSA binding to citrate-coated gold nanoparticles and surfaces. Langmuir 21:9303-9307

29. Lacerda SHDP, Park JJ, Meuse C, Pristinski D, Becker ML, Karim A, Douglas JF (2010) Interaction of gold nanoparticles with common human blood proteins. ACS Nano 4:365-379

30. Lee S-W, Lim J-M, Bhoo S-H, Paik Y-S, Hahn T-R (2003) Colorimetric determination of amino acids using genipin from Gardenia jasminoides. Anal Chim Acta 480:267-274

31. Park J-E, Lee J-Y, Kim H-G, Hahn T-R, Paik Y-S (2002) Isolation and characterization of water-soluble intermediates of blue pigments transformed from geniposide of Gardenia jasminoides. J Agric Food Chem 50:6511-6514

32. Bigi A, Cojazzi G, Panzavolta S, Rubini K, Roveri N (2001) Mechanical and thermal properties of gelatin at different degrees of glutaraldehyde crosslinking. Biomaterials 22:763768

33. Goutam T, Analava M, Rousseau M, Basak A, Sarkar S, Pal K (2011) Crosslinking of gelatin-based drug carriers by genipin induces changes in drug kinetic profiles in vitro. J Mater Sci: Mater Med 22:115-123

34. Li P, Zheng JP, Ma YL, Yao KD (2003) Gelatin/montmorillonite hybrid nanocomposite. II. Swelling behavior. J Appl Polym Sci $88: 322-326$ 
35. Perni S, Piccirillo C, Pratten J, Prokopovich P, Chrzanowski W, Parkin IP, Wilson M (2009) The antimicrobial properties of lightactivated polymers containing methylene blue and gold nanoparticles. Biomaterials 30:89-93

36. Salgueiro AM, Daniel-da-Silva AL, Fateixa S, Trindade T (2013) kCarrageenan hydrogel nanocomposites with release behavior mediated by morphological distinct Au nanofillers. Carbohyd Polym 91:100-109
37. Narband N, Uppal M, Dunnill CW, Hyett G, Wilson M, Parkin IP (2009) The interaction between gold nanoparticles and cationic and anionic dyes: enhanced UV-visible absorption. Phys Chem Chem Phys 11:10513-10518

38. Yunus WMM, Sheng CK, Yunus WMZW (2003) Study on photobleaching of methylene blue doped in PMMA, PVA and gelatin using photoacoustic technique. J Nonlinear Opt Phys 12:91-100 